\title{
Utilisation de capteurs CCD pour l'imagerie et la spectroscopie XUV
}

\section{A. Mens}

CEA, Centre d'Etudes de Limeil-Valenton, 94195 Villeneuve-Saint-Georges cedex, France

Résumé: Les détecteurs CCD classiques sont capables de détecter des photons X d'énergies comprises entre 1 et $10 \mathrm{keV}$; ils ont été utilisés pour cela depuis de nombreuses années, par exemple pour étudier les plasmas créés par laser, dans des appareils tels que les chambres à sténopé ou les spectromètres utilisant un cristal de Bragg en réflexion.

Les senseurs amincis de qualité scientifique ont fait l'objet de développements durant les années 80 et 90 . On rappelle leur principe et on montre que leur structure les rend potentiellement capables de détecter tout rayonnement entre le proche infrarouge et les rayons $\mathrm{X}$ de $10 \mathrm{keV}$, y compris tout le spectre UV, XUV et X mou.

La sensibilité de ces capteurs est bien meilleure que celle des films $X$. Néanmoins, elle est plus faible que celle donnée par les prévisions basées sur l'analyse des constantes optiques du silicium et de la silice. Plusieurs modèles permettent d'expliquer ce phénomène et de quantifier cette perte de sensibilité. Les résultats donnés par l'un d'eux sont présentés ici avec des données expérimentales acquises sur le synchrotron SuperACO, qui nous permettent de connaitre la sensibilité d'un CCD aminci entre $50 \mathrm{eV}$ et $1000 \mathrm{eV}$. Ils confirment que, avec une bonne électronique de lecture, des signaux correspondant à quelques photons par pixel peuvent être détectés.

On montre également les performances et diverses applications expérimentales du système que nous utilisons au CEL-V avec ce type de détecteur. d'évolution.

Enfin, la conclusion en résume l'état de l'art et donne leurs perspectives

\section{Introduction}

Les dispositifs à transfert de charges ont été inventés vers 1970 [1,2] pour servir de lignes à retard analogiques. Par abus de langage, on les désigne en général sous le nom de CCD (Charge Coupled Device, circuit à charge couplée) qui est le type le plus répandu des composants de cette famille. Par suite de leur intérêt comme photodétecteur, et du fait de l'importance des marchés liés à la télévision, leur principal domaine d'application est toutefois rapidement devenu l'imagerie électronique.

Dans les années 70, la technologie ne permettait d'obtenir que des capteurs de performances modestes. Les capteurs utilisés à des fins scientifiques étaient alors souvent les mêmes que ceux des caméras vidéo. La possibilité d'utiliser les CCD dans le domaine $\mathrm{X}$ entre 1 et $10 \mathrm{keV}$ a été démontrée à ce moment [3]. Dans les années 80, les progrès de la technologie ont conduit à une amélioration sensible de leurs performances. Compte tenu de 
besoins très différents, il s'est alors produit une dissociation entre capteurs destinés à la vidéo et capteurs scientifiques. Dans le premier domaine, on a privilégié la recherche de bonnes résolutions mais aussi de capteurs à bas prix, donc de petite taille. Dans le second par contre, on a recherché une grande dynamique et un rendement quantique maximal. Cela s'est traduit par l'utilisation d'étages de sortie optimisés pour un bruit de lecture aussi faible que possible, des pixels restant relativement grands pour avoir une capacité de stockage de charges maximale, une surface photosensible de plus en plus grandes avec l'apparition des capteurs "a image totale" (full frame") ne fonctionnant qu'en monocoup. Enfin, et notamment sous l'impulsion des astrophysiciens[4,5], il a fallu concevoir des structures adaptées à la détection de types de rayonnement donnés, tels que le CCD à phase virtuelle (VPCCD) de Texas Instruments[6], ou les CCD amincis éclairés par l'arrière, fabriqués par plusieurs laboratoires ou industriels, qui ont eu pour intérêt initial d'offrir une efficacité de détection accrue dans l'ultraviolet. En parallèle sont apparus des systèmes d'imagerie mettant en oeuvre ces capteurs scientifiques de façon à en exploiter au mieux les performances et pouvoir ainsi analyser quantitativement et précisément le contenu d'une image.

Cet article étudie la sensibilité des capteurs classiques et amincis dans les domaines $\mathrm{X}$ $(\mathrm{E}<10 \mathrm{keV})$ et $\mathrm{UV}$ et en donne quelques exemples d'utilisation.

\section{Le potentiel des CCD pour la détection $X$ et UV}

Un photodétecteur à l'état solide utilise la propriété des semiconducteurs, en général du silicium, de libérer des charges électrique sous l'influence d'un rayonnement. Ces charges doivent ensuite être soit écoulées si l'on veut une résolution temporelle de l'éclairement, soit stockées dans le photodétecteur. Le signal que celui-ci délivre est alors proportionnel à l'intégrale du flux lumineux qu'il a reçu pendant son temps de stockage, aussi appelé temps d'intégration.Les deux structures utilisées pour cela sont la photodiode et le photoMOS (cf.figure 1). Un CCD est une matrice de photoMOS.

PHOTODIODE

PHOTO MOS

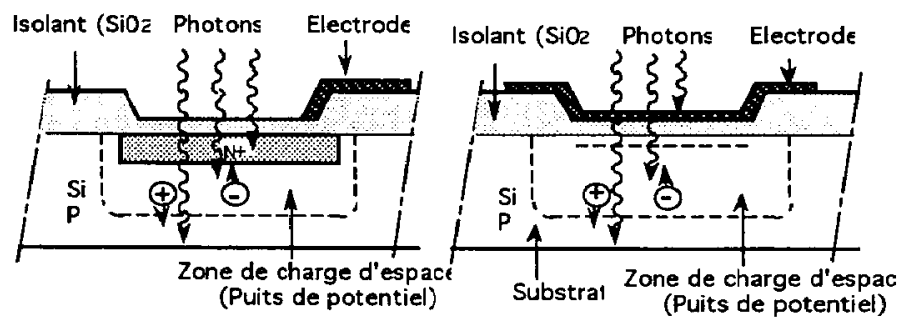

Figure 1: Différents types de photodétecteurs

Les photons incidents, avant de créér une charge dans le volume du semiconducteur, doivent traverser un réseau d'électrodes en silicium et une couche de silice destinée à produire l'effet de champ, dont les épaisseurs respectives sont de plusieurs milliers d'Angströms. De plus le capteur est généralement' recouvert d'une couche de silice supplémentaire épaisse de plus d'un micromètre destinée à sa protection, donc le CCD classique ne sera pas sensible aux rayonnements dont la longueur d'absorption dans la silice et le silicium est de quelques dizaines ou centaines d'Angströms (UV,XUV, X mous). On a donc pensé à enlever le substrat sur lequel est déposé la zone active du détecteur, jusqu'à l'interface avec celle-ci. On peut alors éclairer le CCD aminci par la face arrière et pénètrer directement dans la région où s'effectue la détection, dont l'épaisseur est en général de l'ordre de 10 micromètres; il ne reste plus aux photons qu'à traverser une couche de silice épaisse d'environ une centaine d'Angströms, destiné à la passivation de la face arrière, pour produire un signal, ce qui sera possible dans les gammes spectrales citées ci-dessus. La figures 2 donne les longueurs d'absorption du silicium et de la silice en 
fonction de l'énergie des photons extraites des tables de Henke, et la figure 3 montre l'efficacité de détection comparée des deux structures, qui représente le taux de photons absorbés dans la zone active par rapport aux photons incidents.

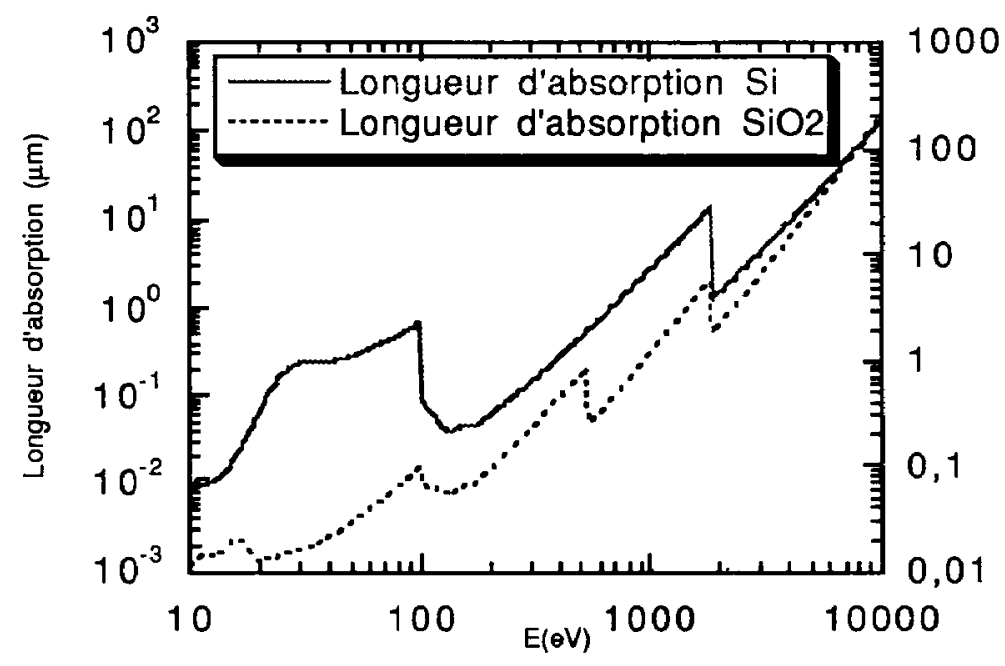

Figure 2

Longueurs d'absorption de $\mathrm{Si}$ et $\mathrm{SiO}_{2}$ en fonction de l'énergie

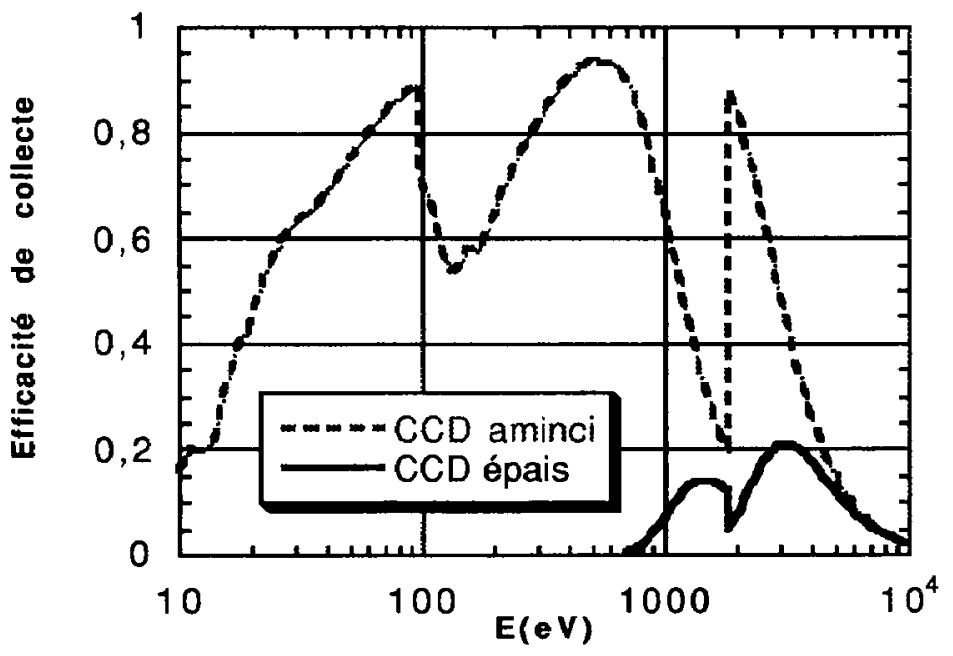

Figure 3

Efficacité de détection comparłe des CCD éclairés par l'avant et l'arrière 


\section{Utilisation des $\mathrm{CCD}$ classiques dans le domaine $\mathrm{X}$}

La courbe donnant l'efficacité de collecte des CCD épais en fonction de l'énergie a été vérifiée expérimentalement de longue date [3]. Dès cette date, ils ont été utilisés dans les diagnostics des plasmas créés par laser en tant que détecteurs dans les chambres à sténopé afin de permettre le pointage des faisceaux laser sur cible.

Au CEL-V, nous avons développé en collaboration avec Thomson CSF L.E.R. et A.R.P. un système d'acquisition d'images à grande dynamique adapté à l'environnement d'une chambre d'expériences [7]. Celui-ci se compose d'une carte d'extension pour PC et d'une caméra à sortie numérique, qui utilise des détecteurs fournis par Thomson TCS. La figure 4, acquise avec ce système sur le laser Nova du LLNL (USA), représente les zones d'interaction de cinq des faisceaux laser avec une cible plane.

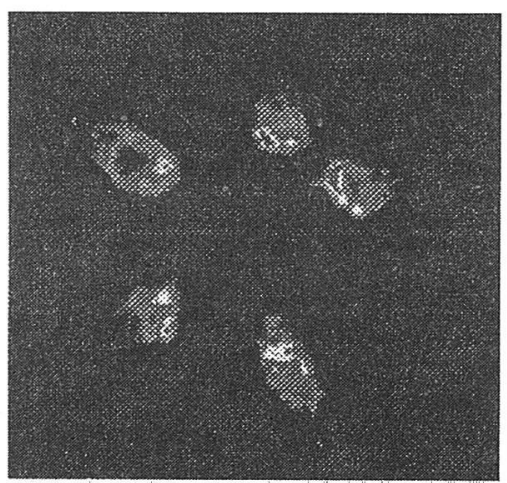

Figure 4: Interaction de faisceaux laser avec une cible plane visualisée par une chambre à sténopé

Dans les plasmas créés par laser, la spectroscopie entre 1 et $10 \mathrm{keV}$ apporte des informations particulièrement intéressantes sur l'état du milieu où ils sont crés. Par conséquent l'adaptation de CCD classiques sur des spectromètres à cristaux de Bragg travaillant dans cette garnme a été la deuxième application notable. La figure 5 représente un spectre d'émission d'une cible de $\mathrm{NaCl}$ analysé par un spectromètre à cristal de TlAP concave conçu au CEL-V [ 8], acquis sur l'expérience Heliotrope.
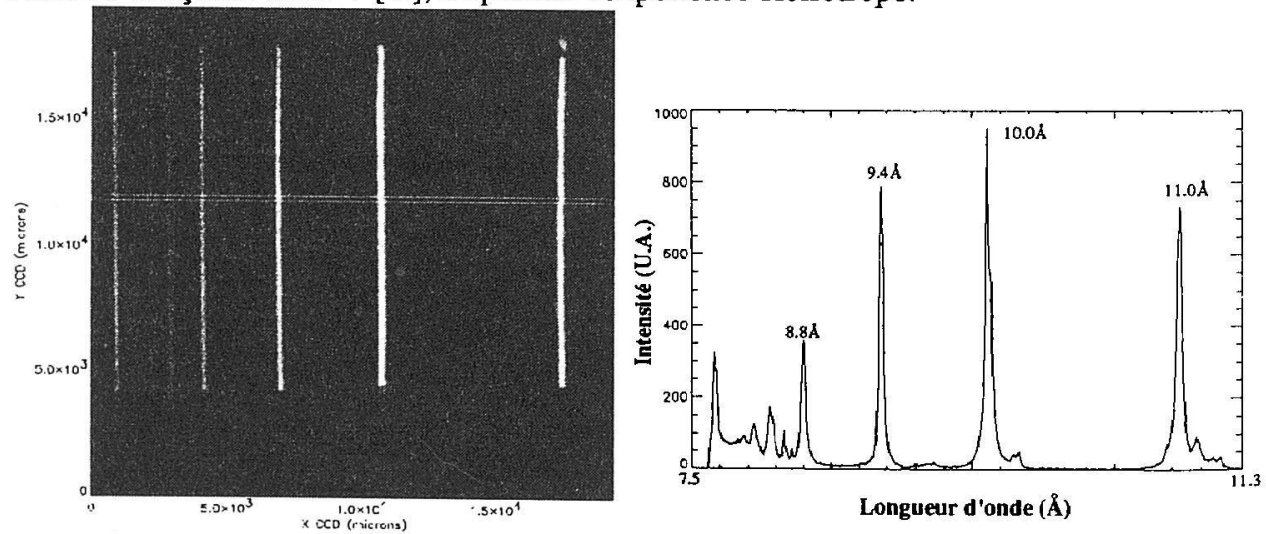

Figure 5: Spectre acquis par un spectromètre à cristal à lecture par CCD 


\section{Performances des CCD amincis dans le domaine $10 \mathrm{eV}-10 \mathrm{keV}$}

La figure 6 montre la structure d'un CCD aminci. L'épaisseur de la couche inactive en face arrière n'est pas, pour certaines gammes d'énergies, négligeable devant la profondeur de pénètration des photons (figure 2). Certaines mesures ont montré que les rendements mesurés dans ces plages sont sensiblement plus faibles que ceux auxquels on pourrait s'attendre: des pièges à l'interface $\mathrm{Si}_{-} \mathrm{SiO}_{2}$ de face arrière tendent à capturer une partie des charges libérées par les photons incidents dans la partie la plus proche du Si. De nombreux modèles développés pour rendre compte de ce phénomène ont été validés dans différentes gammes spectrales $[9,10,11,12,13]$. Un modèle a été conçu au CEL-V, qui part de la résolution des Equations de diffusion des électrons dans un profil de champ électrique simplifié, et dont nous avons comparé les résultats avec des mesures effectuées sur le synchrotron SuperACO. La figure 7 montre le résultat obtenu, et la figure 8 l'influence calculée par le modèle de ce phénomène de recombinaison sur la sensibilité du CCD.

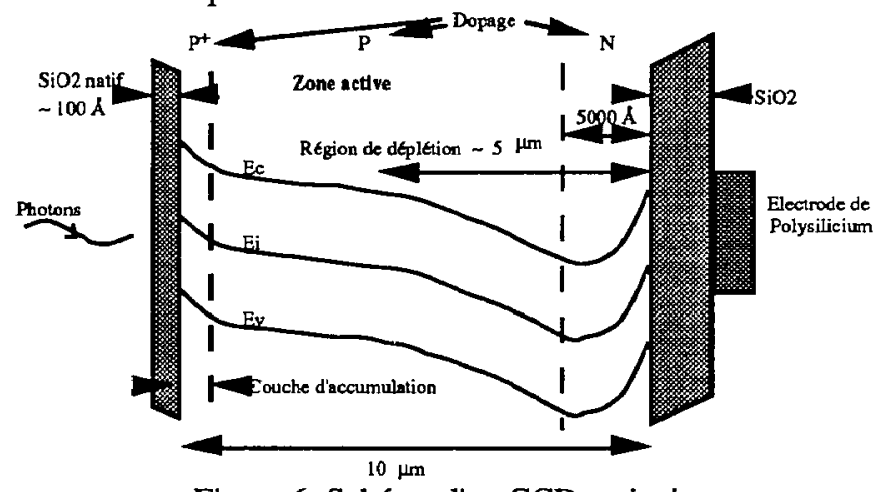

Figure 6: Schéma d'un CCD aminci

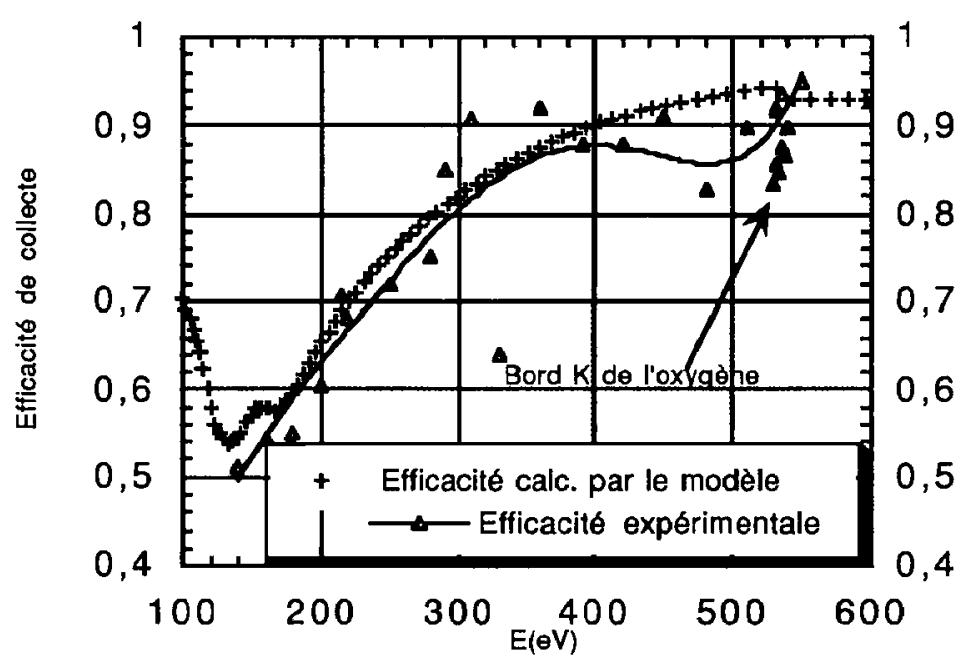

Figure 7: Comparaison modèle-expérience 


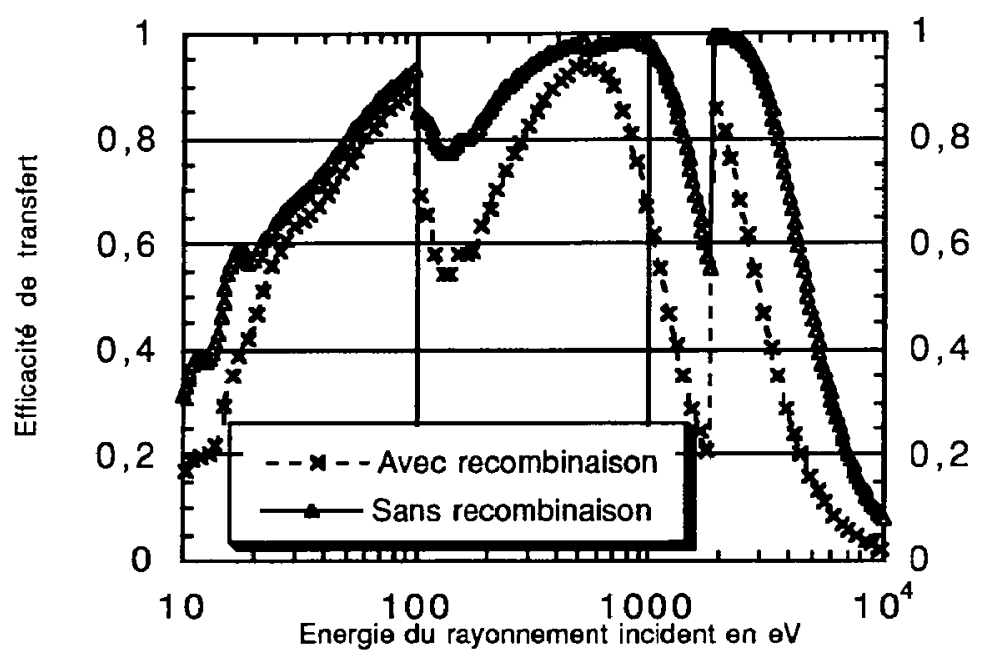

Figure 8: Influence de la recombinaison

On s'aperçoit donc que les $\mathrm{CCD}$ amincis sont malgré ces limitations sensibles dans tout le domaine XUV avec des efficacités quantiques très bonnes. Par rapport aux films $X$ notamment, une grande différence de sensibilité (supérieure à un facteur 100) a toujours pu être constatée à l'avantage du CCD.

\section{Les CCD amincis dans le domaine XUV}

Dans ce domaine également on retrouve des exemples d'utilisation dans l'imagerie et dans la spectroscopie. Ainsi un CCD aminci a été utilisé comme détecteur dans un microscope de Schwarzschild fonctionnant à $60 \mathrm{eV}$ sur l'installation Heliotrope en 1991 [14]. La figure 9 montre la tache de diffraction obtenue dans le plan focal d'un faisceau du laser Octal sur lequel était disposé une lame de phase aléatoire à éléments carrés. On repère très bien la figure dûe à cette structure.

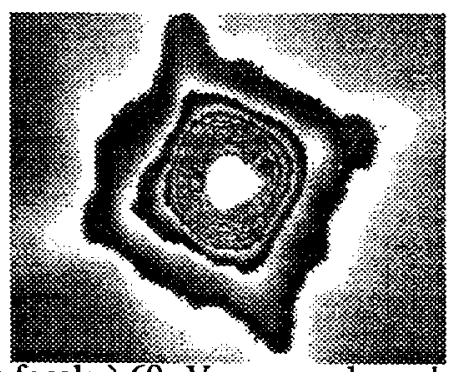

Figure 9: Tache focale a $60 \mathrm{eV}$ avec une lame de phase aléatoire

De même des spectres divers ont pu être acquis en plaçant ces détecteurs derrière des réseaux de diffraction fonctionnant en transmission[14,15]. La figure 10 montre un spectre d'émission d'une cible d'or acquis sur la même installation. On reconnait distintement au centre de l'image l'ordre 0 beaucoup plus intense et de part et d'autre les ordres +1 et -1 . 

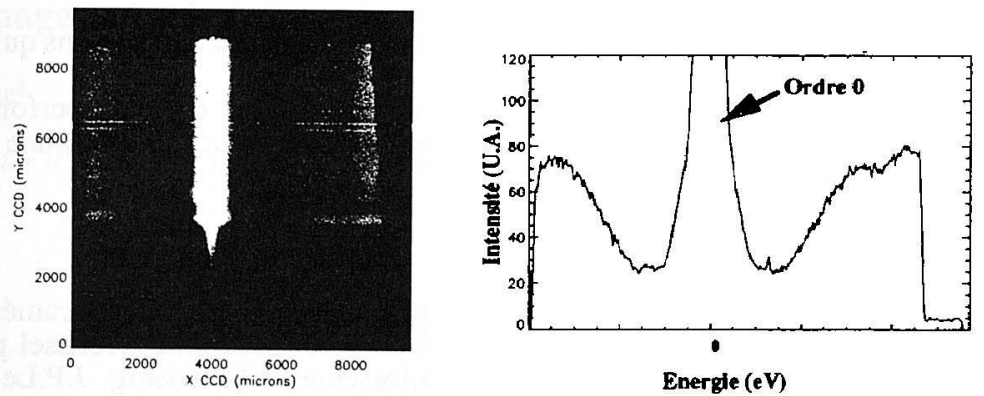

Figure 10: Spectre acquis avec un réseau en transmission associe à un CCD aminci

Une autre application sur les expériences de plasmas crés par laser a consisté, avec l'equipe du LSAI, à utiliser la calibration de la sensibilité du CCD aminci pour quantifier l'énergie émise dans un faisceau laser X émis par une cible de $\mathrm{Zn}$ à $212 \AA$ à l'Ecole Polytechnique.

De plus, lorsque le flux incident est suffisamment faible pour qu'un seul photon au plus soit absorbé dans chaque pixel, il est possible d'effectuer de la spectroscopie non dispersive (les photons sont directement analysés par le CCD sans être préalablement diffractés) à l'aide du CCD: connaissant la quantité de charges liberée par chaque photon, on peut remonter à son énergie: chaque photon d'énergie $E$ absorbé dans la zone de détection libère en moyenne $\mathrm{N}=\mathrm{E}(\mathrm{eV}) / 3.65$ électrons; la résolution en énergie est déterminée par l'incertitude sur la charge libérée autour de cette moyenne dont la variance est égale à F.N où $F$ est une constante appelée facteur de Fano, dont la valeur est voisine de 0.1. Un exemple d'une telle utilisation est donné par des expériences d'étude d'interaction laser ultracourt-cible solide menées au LOA de l'ENSTA par l'équipe du LULI.

Il faut également souligner que ce détecteur peut être utilisé sur d'autres sources de rayonnement XUV en laboratoire (comme l'indique l'article de P.Troussel dans cette revue). Par exemple son utilité sur les expériences synchrotron est évidente; à titre d'exemple on peut dire qu'il permet de caractériser le faisceau dont on dispose sur une ligne: sur la figure 11 on peut observer une image à $480 \mathrm{eV}$ du faisceau délivré sur la ligne SU71 de SuperACO, acquise lors de nos expériences de détermination de la sensibilité du CCD (le faisceau est partiellement occulté par les fentes de collimation du faisceau et par l'obturateur utilisé).

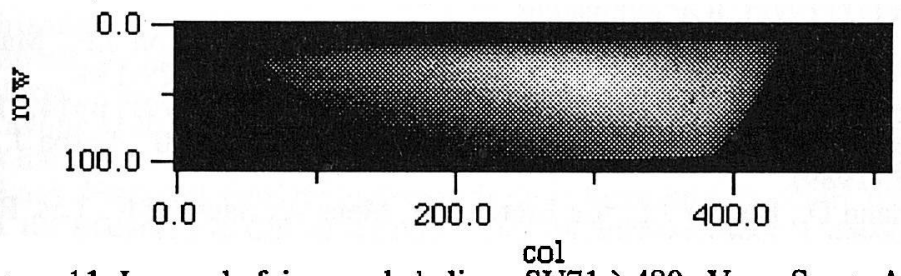

Figure 11: Image du faisceau de la ligne SU71 à $480 \mathrm{eV}$ sur Super ACO

\section{Conclusion}

Les CCD classiques ont été utilisés de longue date pour la détection de rayonnement $X$. Leur utilisation s'accroit au fur et à mesure que leurs performances et celles des systèmes associés progressent.

Les CCD amincis sont maintenant disponibles couramment auprès des fabricants comme le prouvent les exemples d'applications donnés ci-dessus, réalisés avec du matériel cataloguc. D'autres équipes ont intégré une détection par CCD sur des expériences d'imagerie ou de spectroscopie dans le domaine $\mathrm{X}$ en utilisant différentes caméras. Il serait donc impossible 
de faire une liste exhaustive des matériels utilisés et des différentes applications qui ont été réalisées.

La compréhension de leur fonctionnement et la caractérisation de leurs performances permettent maintenant de les utiliser comme d'autre types de détecteurs pour une analyse quantitative de données expérimentales.

\section{Remerciements}

L'auteur remercie D.Mazataud et A.Mugnier pour la caractérisation de la caméra CCD utilisée, J.P.Baudens et P.Salières pour le travail de modélisation, P.Troussel pour les tests sur Super ACO, T.Jalinaud, J.L.Bocher, D.Desenne, J.M.Koenig, J.P.Le Breton pour la mise en oeuvre sur l'installation Heliotrope et la fourniture d'images expérimentales, L.Da Silva (LLNL) pour une contribution analogue sur la chambre d'expériences Nova à Livermore ainsi que des discussions fructueuses sur la caractérisation et l'utilisation de ces capteurs, B.Rus, P.Goedtkindt et leurs collègues du LSAI pour la mesure sur l'expérience Laser X, A.Rousse, P.Audebert et J.P.Geindre du LULI pour les discussions sur l'utilisation du CCD en spectroscopie non dispersive.

\section{Références}

[1] Sangster F.L.J. et Teer K., IEEE J.Solid State Circ., vol. SC-4 ( juin 1969) pp131136.

[2] Boyle W.S. et Smith G.E., Bell Syst. Tech. J., vol.49 (1970) pp487-493.

[3] Koppel L.N., Rev. Sci. Instrum. 48 (6) (1977) pp669-672.

[4] Janesick J.R., Elliott T., Marsh H.H., Collins S., McCarthy J., Blouke M., Rev. Sci. Instrum. 56 (5) (1985) pp796-801.

[5] Marsh H.H., Joshi C., Janesick J., Collins S., Rev. Sci. Instrum. 56 (5) (1985) pp837-839.

[6] Stern R.A., Liewer K., Janesick J.R., Rev. Sci. Instrum. 54 (2) (1983) pp198-205.

[7] Mens A., Ducrocq N., Mazataud D., Mugnier A., Eouzan J.Y., Heurtaux J.C., Tomasini F., Mathae J.C., SPIE vol. 1358 (1990) pp719-731.

[8] Combis P., Desenne D., Louisjacquet M., à paraître dans Rev. Sci. Instrum.

[9] Bailey P., Castelli C., Cross M., Van Essen P., Holland A., Jansen F., De Korte P., Lumb P., Mc Carthy K., Pool P., Verhoeve P., Proc. EUV, X-ray and Gamma-ray Instr. for astronomy and atomic physics, SPIE (1983).

[10] Salières P., Mens A., Mazataud D., Schirmann D., Benattar R., Inst. Phys. conf. Ser. No 125 (7) (1992), IOP Publishing.

[11] Mens A., Benattar R., Bocher J.L., Koenig J.M., Le Breton J.P., Mazataud D., Salières P., Schirmann D., J.Optics (Paris), Vol.24 (3)(1993) pp129-134.

[12] Blouke M., Delamere W.A., Womack G., SPIE vol. 1447 (1991) pp142-154.

[13] Hochedez J.F., Lemaire P., Delaboudinière J., Cougrand B., Barba J., proc. OE Laser, SPIE (1989).

[14] Schirmann D., Bocher J.L., Le Breton J.P., Mens A., Sauneuf R., Las. Part. Beams vol.10 (4) (1992) pp827-839.

[15] Pina L., Fiedorowicz H., Koshevoi M.O., Rupasov A.A., Rus B., Shikanov A., Svoboda V., Las. Part. Beams, vol. 9 (2) (1991) pp579-591. 